\title{
EQUILIBRIUM POINTS FOR A SYSTEM INVOLVING $m$-ACCRETIVE OPERATORS
}

\author{
C. E. CHIDUME ${ }^{1}$ AND M. O. OSILIKE ${ }^{2}$ \\ ${ }^{1}$ The Abdus Salam International Centre for Theoretical Physics, \\ PO Box 586, 34100 Trieste, Italy \\ ${ }^{2}$ Department of Mathematics, University of Nigeria, Nsukka, Nigeria
}

(Received 27 October 1998)

\begin{abstract}
Let $E$ be a real uniformly smooth Banach space and let $A$ be a nonlinear $\phi$-strongly quasiaccretive operator with range $R(A)$ and open domain $D(A)$ in $E$. For a given $f \in E$, let $A$ satisfy the evolution system $\mathrm{d} u(t) / \mathrm{d} t+A u(t)=f, u(0)=u_{0}$. We establish the strong convergence of the Ishikawa and Mann iterative methods with appropriate error terms recently introduced by Xu to the equilibrium points of this system. Related results deal with the strong convergence of the iterative methods to the fixed points of $\phi$-strong pseudocontractions defined on open subsets of $E$.
\end{abstract}

Keywords: uniformly smooth spaces; accretive operators; pseudocontractive operators; Ishikawa and Mann iterative methods; fixed points; equilibrium points

AMS 2000 Mathematics subject classification: Primary 47H06; 47H15; 47H17

\section{Introduction and preliminaries}

Let $E$ be a real normed space. A mapping $U$ with domain $D(U)$ and range $R(U)$ in $E$ is called accretive if the inequality

$$
\|x-y\| \leqslant\|x-y+s(U x-U y)\|
$$

holds for every $x, y \in D(U)$ and for all $s>0$. For a Banach space $E$ we shall denote by $J$ the normalized duality map from $E$ to $2^{E^{*}}$ defined by $J x=\left\{f^{*} \in E^{*}:\left\langle x, f^{*}\right\rangle=\|x\|^{2}=\right.$ $\left.\left\|f^{*}\right\|^{2}\right\}$, where $E^{*}$ denotes the dual space of $E$ and $\langle\cdot, \cdot\rangle$ denotes the generalized duality pairing. As a consequence of a result of Kato [24], the accretive condition (1.1) can be expressed in terms of the normalized duality map as follows. For each $x, y \in D(U)$, there exists $j(x-y) \in J(x-y)$ such that $\langle U x-U y, j(x-y)\rangle \geqslant 0$. A mapping $T: D(T) \subseteq E \rightarrow E$ is called strongly accretive if for all $x, y \in D(T)$ there exist a constant $k>0$ and $j(x-y) \in J(x-y)$ such that $\langle T x-T y, j(x-y)\rangle \geqslant k\|x-y\|^{2}$. The map $T$ is called $\phi$-strongly accretive if there exist $j(x-y) \in J(x-y)$ and a strictly increasing function $\phi:[0, \infty) \rightarrow[0, \infty)$ with $\phi(0)=0$ such that for each $x, y \in E$ :

$$
\langle T x-T y, j(x-y)\rangle \geqslant \phi(\|x-y\|)\|x-y\| .
$$


The operator $T$ is called $m$-accretive if it is accretive and $(I+r T)(D(T))=E$ for all $r>0$, where $I$ denotes the identity operator on $D(T)$. Moreover, if the nullspace of $T$, $N(T):=\{x \in D(T): T x=0\}$, is non-empty and the relations (1.1) and (1.2) hold for any $x \in D(T)$ but $y \in N(T)$, then the corresponding operator $T$ is called quasi-accretive, quasi $\phi$-strongly accretive, respectively.

Closely related to the class of accretive operators is the class of pseudocontractive operators. An operator $T$ with domain $D(T)$ and range $R(T)$ in $E$ is called strongly pseudocontractive if for all $x, y \in D(T)$, there exist $j(x-y) \in J(x-y)$ and a constant $t>1$ such that $\langle T x-T y, j(x-y)\rangle \leqslant t^{-1}\|x-y\|^{2}$. If $t=1$, then $T$ is called pseudocontractive. The map $T$ is called $\phi$-strongly pseudocontractive if for all $x, y \in D(T)$, there exist $j(x-y) \in J(x-y)$ and a strictly increasing function $\phi:[0, \infty) \rightarrow[0, \infty)$ with $\phi(0)=0$ such that

$$
\langle T x-T y, j(x-y)\rangle \leqslant\|x-y\|^{2}-\phi(\|x-y\|)\|x-y\| .
$$

$T$ is called $\phi$-hemicontractive if relation (1.3) holds for all $x \in D(T)$ and $x^{*} \in N(I-T)$. It follows from inequalities (1.2) and (1.3) that $T$ is $\phi$-strongly pseudocontractive if and only if $(I-T)$ is $\phi$-strongly accretive, so that the mapping theory for accretive operators is intimately connected with the fixed-point theory for pseudocontractions.

The notion of accretive operators was introduced in 1967 by Browder [1] and Kato [24]. An early fundamental result in the theory of accretive operators, due to Browder, states that the initial-value problem

$$
\frac{\mathrm{d} u}{\mathrm{~d} t}+A u=0, \quad u(0)=u_{0},
$$

is solvable if $A$ is locally Lipschitzian and accretive on $E$. This result was subsequently generalized by Martin [28] to the continuous accretive operators. It is well known (see, for example, $[\mathbf{1 0}, \mathbf{3 9}])$ that many physically significant problems can be modelled in terms of an initial-value problem of the form (1.4), where $T$ is either accretive, $\phi$-strongly accretive or $\phi$-strongly quasi-accretive. Typical examples of how such evolution equations arise are found in models involving either the heat, the wave or the Schrödinger equation. If $u$ is independent of $t$,

$$
A u=0,
$$

and the solution of this equation corresponds to the equilibrium points of system (1.4). Consequently, considerable research effort has been devoted, especially within the last 10 years or so, to developing constructive techniques for the determination of the kernels of accretive operators in Banach spaces (see, for example, $[\mathbf{2}, \mathbf{3}, \mathbf{5}-\mathbf{2 3}, \mathbf{2 5}, \mathbf{2 6}, \mathbf{2 9}-\mathbf{3 3}, \mathbf{3 5}, \mathbf{3 7}$, 39-41]).

Two well-known iteration schemes - the Mann iteration scheme (see, for example, $[\mathbf{2 7}]$ ) and the Ishikawa iteration scheme (see, for example, $[\mathbf{2 3}]$ ) - have successfully been employed to approximate the kernels of accretive self-maps. However, if a map $T$ is such that its range does not intersect its domain (and this occurs frequently in many applications), then neither the Mann nor the Ishikawa scheme may be well defined. In fact, 
equation (1.5) may not even have a solution. Take for example $T:[0,1] \rightarrow \mathbb{R}$ defined by $T x=\frac{1}{2} x+1$. Clearly, $T$ is a contraction map but has no fixed point in $[0,1]$. Hence $A:=(I-T):[0,1] \rightarrow \mathbb{R}$ is strongly accretive and $A u=0$ has no solution. In general, if $D(A) \cap R(A)=\emptyset$, some extra condition on $A$ must be imposed for equation (1.5) to have a solution. One such condition which has been studied extensively and for which fixed-point theorems have been established is that of 'weak inwardness' (see, for example, [4]). For weakly inward maps no generality is lost if the domain is assumed to be an open set (see, for example, $[\mathbf{4}, \mathrm{ch}$. IV]). In $[\mathbf{2}]$, Bruck studied the problem of iteratively approximating the solution of $A u=f$ for a given $f \in H$, where $A:=(I+T)$ and $T: H \rightarrow H$ is a monotone operator on a Hilbert space $H$. (In Hilbert spaces accretive operators are called monotone.) He proved the following theorem.

Theorem B (see page 1259 in [2]). Let $T$ be a multivalued monotone operator with open domain $D(T)$ in a Hilbert space $H$ and $f \in R(I+T)$. Then there exist a neighbourhood $N \subseteq D(T)$ of $x^{*}=(I+T)^{-1} f$ and a real number $\sigma_{1}>0$ such that for any $\sigma \geqslant \sigma_{1}$, any initial guess $x_{1} \in N$ and any single-valued section $T_{0}$ of $T$, the sequence $\left\{x_{n}\right\}$ generated from $x_{1}$ by

$$
x_{n+1}=x_{n}-(n+\sigma)^{-1}\left(x_{n}+T_{0} x_{n}-f\right)
$$

remains in $D(T)$ and converges to $x^{*}$ with $\left\|x_{n}-x^{*}\right\|=O\left(n^{-1 / 2}\right)$.

Let $E$ be a real Banach space. The modulus of smoothness of $E$ is the function $\rho_{E}$ : $[0, \infty) \rightarrow[0, \infty)$ defined by

$$
\rho_{E}(\tau):=\frac{1}{2} \sup \{\|x+y\|+\|x-y\|-2:\|x\| \leqslant 1,\|y\| \leqslant \tau\} .
$$

For $q>1, E$ is called $q$-uniformly smooth if there exists a constant $c>0$ such that $\rho_{E}(\tau) \leqslant c \tau^{q}$, and is called uniformly smooth if

$$
\lim _{\tau \rightarrow 0} \frac{\rho_{E}(\tau)}{\tau}=0
$$

Clearly, every $q$-uniformly smooth Banach space is uniformly smooth. Moreover, it is well known (see, for example, $[\mathbf{3 6}, \mathbf{3 8}]$ ) that Hilbert spaces are 2-uniformly smooth, while

$$
L_{p}\left(\text { or } \ell_{p}\right) \quad \text { or } \quad W_{m}^{p} \text { is } \begin{cases}p \text {-uniformly smooth } & \text { if } 1<p \leqslant 2 \\ 2 \text {-uniformly smooth } & \text { if } p \geqslant 2 .\end{cases}
$$

Since its publication in 1973, several authors have extended Theorem B to more general Banach spaces (see, for example, $[\mathbf{1 0}, \mathbf{1 1}, \mathbf{1 3}, \mathbf{1 5}, \mathbf{4 0}]$ ). The most general result of type Theorem B now known appears to be the following recent theorem of one of the authors.

Theorem C (see Theorem 4.1 on page 56 in [11]). Let $E$ be a real $q$-uniformly smooth Banach space, $q>1$. Suppose $A$ is a set-valued strongly accretive operator with open domain $D(A)$ in $E$ and $f \in A x$ has a solution $x^{*} \in D(A)$. Then there exist a neighbourhood $B \subseteq D(A)$ of $x^{*}$ and real number $r_{1}>0$ such that for any $r>r_{1}$, any 
initial guess $x_{1} \in B$, any single-valued selection $A_{0}$ of $A$ and some real sequence $\left\{c_{n}\right\}_{n=1}^{\infty}$, the sequence $\left\{x_{n}\right\}_{n=1}^{\infty}$ generated from $x_{1}$ by

$$
x_{n+1}=x_{n}+c_{n}\left(f-A_{0} x_{n}\right), \quad n \geqslant 1,
$$

remains in $D(A)$ and converges strongly to $x^{*}$ with $\left\|x_{n}-x^{*}\right\|=O\left(n^{(q-1) / q}\right)$.

In 1995, Liu [25] first introduced what he called Ishikawa and Mann iteration processes 'with errors' for nonlinear strongly accretive mappings. Recently, Yuguang Xu [37] objected to the definition of Liu (see [37]) and then introduced the following definitions.

(A) Let $K$ be a non-empty convex subset of $E$ and $T: K \rightarrow K$ a mapping. For any given $x_{0} \in K$, the sequence $\left\{x_{n}\right\}$, defined iteratively by

$$
x_{n+1}=a_{n} x_{n}+b_{n} T y_{n}+c_{n} u_{n} ; \quad y_{n}=a_{n}^{\prime} x_{n}+b_{n}^{\prime} T x_{n}+c_{n}^{\prime} v_{n}, \quad n \geqslant 0,
$$

where $\left\{u_{n}\right\},\left\{v_{n}\right\}$ are bounded sequences in $K$, and $\left\{a_{n}\right\},\left\{b_{n}\right\},\left\{c_{n}\right\},\left\{a_{n}^{\prime}\right\},\left\{b_{n}^{\prime}\right\}$, $\left\{c_{n}^{\prime}\right\}$ are sequences in $(0,1)$ such that $a_{n}+b_{n}+c_{n}=1=a_{n}^{\prime}+b_{n}^{\prime}+c_{n}^{\prime}$ for all integers $n \geqslant 0$, is called the Ishikawa iteration sequence with errors.

(B) If, with the same notation and definitions as in (A), $b_{n}^{\prime}=c_{n}^{\prime}=0$ for all integers $n \geqslant$ 0 , then the sequence $\left\{x_{n}\right\}$, now defined by $x_{0} \in K, x_{n+1}=a_{n} x_{n}+b_{n} T x_{n}+c_{n} u_{n}$, $n \geqslant 0$, is called the Mann iteration sequence with errors.

It is our purpose in this paper to consider iteration processes of the Ishikawa and Mann types with errors in the sense of Yuguang [37] and prove theorems much more general than Theorem $\mathrm{C}$ in the sense that our theorems hold for the much more general class of $\phi$-strongly accretive operators and in the more general real uniformly smooth spaces. Moreover, our method is of independent interest.

\section{Main results}

In the sequel we shall need the following lemma.

Lemma $\mathbf{X}-\mathbf{R}$ (see $[\mathbf{3 8}, \mathbf{3 9}]$ ). Let $E$ be a real uniformly smooth Banach space. Then, for every $x, y \in E$, and some positive constants $C$ and $D$, we have

$$
\|x+y\|^{2} \leqslant\|x\|^{2}+2\langle y, j(x)\rangle+D \max \left\{\|x\|+\|y\|, \frac{1}{2} C\right\} \rho_{E}(\|y\|) .
$$

\subsection{Convergence theorems for $\Phi$-strongly accretive operator equations}

Theorem 2.1. Let $E$ be a real uniformly smooth Banach space and let $T: D(T) \subseteq$ $E \rightarrow E$ be a $\phi$-strongly accretive operator with open domain $D(T) \subseteq E$. Suppose the equation $T x=f$ has a solution $x^{*} \in D(T)$ for a given $f \in E$. Define $S: D(T) \rightarrow E$ by $S x=x-T x+f$. Then there exist a real number $\mu \geqslant 1$ and a neighbourhood $B \subseteq D(T)$ of $x^{*}$ such that starting with arbitrary $x_{0}, u_{0}, v_{0} \in B$, the sequence $\left\{x_{n}\right\}_{n=0}^{\infty}$ defined iteratively by

$$
\begin{aligned}
y_{n} & =a_{n} x_{n}+b_{n} S x_{n}+c_{n} u_{n}, \quad n \geqslant 0, \\
x_{n+1} & =a_{n}^{\prime} x_{n}+b_{n}^{\prime} S y_{n}+c_{n}^{\prime} v_{n}, \quad n \geqslant 0,
\end{aligned}
$$


remains in $B$ and converges strongly to $x^{*}$, where

(i) $a_{n}+b_{n}+c_{n}=a_{n}^{\prime}+b_{n}^{\prime}+c_{n}^{\prime}=1$;

(ii) $b_{n}=b_{n}^{\prime}=1 /(2(\mu+n)), n \geqslant 0$;

(iii) $c_{n}=c_{n}^{\prime}=1 /\left(2(n+\mu)^{2}\right), n \geqslant 0$; and

(iv) $\left\{u_{n}\right\}$ and $\left\{v_{n}\right\}$ are arbitrary bounded sequences in $E$.

Proof. Set $\beta_{n}=b_{n}+c_{n}, \alpha_{n}=b_{n}^{\prime}+c_{n}^{\prime}$, then (2.2) and (2.3) become

$$
\begin{aligned}
y_{n} & =\left(1-\beta_{n}\right) x_{n}+\beta_{n} S x_{n}+c_{n}\left(u_{n}-S x_{n}\right), \quad n \geqslant 0, \\
x_{n+1} & =\left(1-\alpha_{n}\right) x_{n}+\alpha_{n} S y_{n}+c_{n}^{\prime}\left(v_{n}-S y_{n}\right), \quad n \geqslant 0 .
\end{aligned}
$$

Observe that $x^{*}$ is a fixed point of $S$ and that $(I-S)$ is accretive and, hence, is locally bounded at each interior point of its effective domain. Choose $r>0$ such that $B=$ $\bar{B}_{r}\left(x^{*}\right)=\left\{x \in E:\left\|x-x^{*}\right\| \leqslant r\right\}$ is contained in $D(T)=D(S)$, and $(I-S)(B)$ is bounded. Let $d:=\operatorname{diam}[(I-S)(B)]$, and $M_{1}=\max \left\{\sup \left\|u_{n}-x^{*}\right\|, \sup \left\|v_{n}-x^{*}\right\|\right\}$. Then, for all $x \in B$,

$$
\left\|u_{n}-S x\right\| \leqslant\left\|u_{n}-x^{*}\right\|+\left\|x-x^{*}\right\|+\|x-S x\| \leqslant M_{1}+r+d .
$$

Similarly, $\left\|v_{n}-S x\right\| \leqslant M_{1}+r+d$. Set $d^{*}:=2(r+d)+M_{1}, M=D \max \left\{d^{*}, \frac{1}{2} C\right\}$, where $D$ and $C$ are the constants appearing in inequality (2.1). Since $E$ is uniformly smooth,

$$
\frac{\rho_{E}\left(d^{*} \tau\right)}{\tau} \rightarrow 0 \text { as } \tau \rightarrow 0 .
$$

Thus we can choose $\tau_{0}>0$ such that for all $0<\tau<\tau_{0}$, we have

$$
\frac{\rho_{E}\left(d^{*} \tau\right)}{\tau} \leqslant \frac{\phi\left(\frac{1}{2} r\right) r^{2}}{3 M(1+\phi(r)+r)} .
$$

Furthermore, the uniform continuity of $j$ on bounded subsets of $E$ implies that given

$$
\epsilon:=\frac{\phi\left(\frac{1}{2} r\right) r^{2}}{6 d(1+\phi(r)+r)}
$$

we can choose $\delta_{\epsilon}>0$ such that $\|x-y\|<\delta_{\epsilon}$ implies $\|j(x)-j(y)\| \leqslant \epsilon$. Choose $\delta_{0}>0$ such that $\delta_{0}<\delta_{\epsilon}$; define

$$
\mu:=\max \left\{1, \frac{1}{\tau_{0}}, \frac{8 d^{*}}{r}, \frac{d^{*}}{\delta_{0}}, \frac{24 d^{*}(1+\phi(r)+r)}{\phi\left(\frac{1}{2} r\right) r}\right\}
$$

and generate the sequence $\left\{x_{n}\right\}$ iteratively by (2.4) and (2.5). We show that $\left\{x_{n}\right\}$ is well defined and is in $B$. To do this we first prove that $y_{n} \in B$ whenever $x_{n} \in B$. Suppose, for 
contradiction, that there exists $n \geqslant 0$ such that $x_{n} \in B$ and $y_{n} \notin B$. Then $\left\|x_{n}-x^{*}\right\| \leqslant r$ and $\left\|y_{n}-x^{*}\right\|>r$. Hence

$$
\begin{aligned}
\left\|x_{n}-x^{*}\right\| & \geqslant\left\|y_{n}-x^{*}\right\|-\beta_{n}\left\|x_{n}-S x_{n}\right\|-c_{n}\left\|u_{n}-S x_{n}\right\| \\
& >r-2 d^{*} \beta_{n} \quad\left(\text { since }\left\|x_{n}-S x_{n}\right\| \leqslant d^{*},\left\|u_{n}-S x_{n}\right\| \leqslant d^{*} \text { and } c_{n} \leqslant \beta_{n}\right) \\
& \geqslant r-\frac{2 d^{*}}{\mu} \geqslant \frac{1}{2} r
\end{aligned}
$$

so that $\phi\left(\left\|x_{n}-x^{*}\right\|\right)>\phi\left(\frac{1}{2} r\right)$. Since $T$ is $\phi$-strongly accretive, inequality (1.2) implies that for all $x, y \in D(T)$, there exists $j(x-y) \in J(x-y)$ such that

$$
\begin{aligned}
\langle(I-S) x-(I-S) y, j(x-y)\rangle & \geqslant \phi(\|x-y\|)\|x-y\| \\
& \geqslant \frac{\phi(\|x-y\|)}{1+\phi(\|x-y\|)+\|x-y\|}\|x-y\|^{2} \\
& =r(x, y)\|x-y\|^{2},
\end{aligned}
$$

where

$$
r(x, y)=\frac{\phi(\|x-y\|)}{1+\phi(\|x-y\|)+\|x-y\|} \in[0,1), \quad \forall x, y \in D(T) .
$$

Using (2.6) and inequality (2.1) we obtain

$$
\begin{aligned}
\left\|y_{n}-x^{*}\right\|^{2}= & \left\|x_{n}-x^{*}-\beta_{n}\left(x_{n}-S x_{n}\right)+c_{n}\left(u_{n}-S x_{n}\right)\right\|^{2} \\
\leqslant & \left\|x_{n}-x^{*}\right\|^{2}-2 \beta_{n}\left\langle x_{n}-S x_{n}, j\left(x_{n}-x^{*}\right)\right\rangle+2 c_{n}\left\langle u_{n}-S x_{n}, j\left(x_{n}-x^{*}\right)\right\rangle \\
& \quad+D \max \left\{\left\|x_{n}-x^{*}\right\|+\left\|\beta_{n}\left(x_{n}-S x_{n}\right)+c_{n}\left(u_{n}-S x_{n}\right)\right\|, \frac{1}{2} C\right\} \\
& \quad \times \rho_{E}\left(\left\|\beta_{n}\left(x_{n}-S x_{n}\right)+c_{n}\left(u_{n}-S x_{n}\right)\right\|\right) \\
\leqslant & {\left[1-2 \beta_{n} r\left(x_{n}, x^{*}\right)\right]\left\|x_{n}-x^{*}\right\|^{2}+\frac{2 \beta_{n} d^{*} r}{(\mu+n+1)}+D \max \left\{d^{*}, \frac{1}{2} C\right\} \rho_{E}\left(d^{*} \beta_{n}\right) } \\
\leqslant & {\left[1-\beta_{n} r\left(x_{n}, x^{*}\right)\right]\left\|x_{n}-x^{*}\right\|^{2}+\frac{2 d^{*} r \beta_{n}}{(\mu+n+1)}+M \beta_{n} \frac{\rho_{E}\left(d^{*} \beta_{n}\right)}{\beta_{n}} } \\
\leqslant & {\left[1-\beta_{n} r\left(x_{n}, x^{*}\right)\right] r^{2}+2 \frac{d^{*} r \beta_{n}}{\mu}+M \beta_{n} \frac{\rho_{E}\left(d^{*} \beta_{n}\right)}{\beta_{n}}, }
\end{aligned}
$$

since $\beta_{n} r\left(x_{n}-x^{*}\right) \in[0,1)$. Since $\frac{1}{2} r<\left\|x_{n}-x^{*}\right\| \leqslant r$, we have

$$
r\left(x_{n}, x^{*}\right)=\frac{\phi\left(\left\|x_{n}-x^{*}\right\|\right)}{\left(1+\phi\left(\left\|x_{n}-x^{*}\right\|+\left\|x_{n}-x^{*}\right\|\right)\right.} \geqslant \frac{\phi\left(\frac{1}{2} r\right)}{(1+\phi(r)+r)},
$$

so that inequality (2.7) implies

$$
r^{2}<r^{2}-\beta_{n}\left[\frac{\phi\left(\frac{1}{2} r\right) r^{2}}{(1+\phi(r)+r)}-\frac{2 d^{*} r}{\mu}-M \frac{\rho_{E}\left(d^{*} \beta_{n}\right)}{\beta_{n}}\right] \leqslant r^{2},
$$

a contradiction, so that $y_{n} \in B$. We now prove that $x_{n} \in B, \forall n \geqslant 0$. The proof is by induction. By our choice $x_{0} \in B$. Suppose $x_{n} \in B$. Then $y_{n} \in B$. We prove that 
$x_{n+1} \in B$. Assume for contradiction that $x_{n+1} \notin B$. Then $\left\|x_{n+1}-x^{*}\right\|>r$. Thus we have the following estimates,

$$
\begin{aligned}
\left\|x_{n}-x^{*}\right\| & \geqslant\left\|x_{n+1}-x^{*}\right\|-\alpha_{n}\left\|x_{n}-S y_{n}\right\|-c_{n}^{\prime}\left\|v_{n}-S y_{n}\right\| \\
& >r-2 d^{*} \alpha_{n} \quad\left(\text { since } c_{n}^{\prime} \leqslant \alpha_{n},\left\|x_{n}-S y_{n}\right\| \leqslant d^{*} \text { and }\left\|v_{n}-S y_{n}\right\| \leqslant d^{*}\right),
\end{aligned}
$$

and

$$
\begin{aligned}
\left\|y_{n}-x^{*}\right\| & \geqslant\left\|x_{n}-x^{*}\right\|-\beta_{n}\left\|x_{n}-S x_{n}\right\|-c_{n}\left\|u_{n}-S x_{n}\right\| \\
& \geqslant r-2 d^{*} \alpha_{n}-2 d^{*} \alpha_{n}=r-4 d^{*} \alpha_{n} \geqslant \frac{1}{2} r,
\end{aligned}
$$

so that $\phi\left(\left\|y_{n}-x^{*}\right\|\right)>\phi\left(\frac{1}{2} r\right)$. Thus,

$$
\begin{aligned}
& \left\|x_{n+1}-x^{*}\right\|^{2} \\
& =\left\|x_{n}-x^{*}-\alpha_{n}\left(x_{n}-S y_{n}\right)+c_{n}^{\prime}\left(v_{n}-S y_{n}\right)\right\|^{2} \\
& \leqslant\left\|x_{n}-x^{*}\right\|^{2}-2 \alpha_{n}\left\langle x_{n}-S y_{n}, j\left(x_{n}-x^{*}\right)\right\rangle+2 c_{n}^{\prime}\left\langle v_{n}-S y_{n}, j\left(x_{n}-x^{*}\right)\right\rangle \\
& \quad+D \max \left\{\left\|x_{n}-x^{*}\right\|+\left\|\alpha_{n}\left(x_{n}-S y_{n}\right)+c_{n}^{\prime}\left(v_{n}-S y_{n}\right)\right\|, \frac{1}{2} C\right\} \\
& \quad \times \rho_{E}\left(\left\|\alpha_{n}\left(x_{n}-S y_{n}\right)+c_{n}^{\prime}\left(v_{n}-S y_{n}\right)\right\|\right. \\
& \leqslant\left\|x_{n}-x^{*}\right\|^{2}-2 \alpha_{n}\left\langle x_{n}-y_{n}, j\left(x_{n}-x^{*}\right)\right\rangle \\
& \quad-2 \alpha_{n}\left\langle y_{n}-S y_{n}, j\left(x_{n}-x^{*}\right)\right\rangle+\frac{2 \alpha_{n} d^{*} r}{(\mu+n+1)}+M \rho_{E}\left(d^{*} \alpha_{n}\right) \\
& \leqslant\left\|x_{n}-x^{*}\right\|^{2}-2 \alpha_{n} \beta_{n} \phi\left(\left\|x_{n}-x^{*}\right\|\right)\left\|x_{n}-x^{*}\right\|+2 c_{n} \alpha_{n}\left\langle u_{n}-S x_{n}, j\left(x_{n}-x^{*}\right)\right\rangle \\
& \quad-2 \alpha_{n}\left\langle y_{n}-S y_{n}, j\left(x_{n}-x^{*}\right)\right\rangle+\frac{2 \alpha_{n} d^{*} r}{(\mu+n+1)}+M \alpha_{n} \frac{\rho_{E}\left(d^{*} \alpha_{n}\right)}{\alpha_{n}} .
\end{aligned}
$$

Observe that

$$
\begin{aligned}
\left\langle y_{n}-S y_{n}, j\left(x_{n}-x^{*}\right)\right\rangle & =\left\langle y_{n}-S y_{n}, j\left(x_{n}-x^{*}\right)-j\left(y_{n}-x^{*}\right)\right\rangle+\left\langle y_{n}-S y_{n}, j\left(y_{n}-x^{*}\right)\right\rangle \\
& \geqslant r\left(y_{n}, x^{*}\right)\left\|y_{n}-x^{*}\right\|^{2}+\left\langle y_{n}-S y_{n}, j\left(x_{n}-x^{*}\right)-j\left(y_{n}-x^{*}\right)\right\rangle . \\
\left\|y_{n}-x^{*}\right\| & =\left\|x_{n}-x^{*}-\beta_{n}\left(x_{n}-S x_{n}\right)+c_{n}\left(u_{n}-S x_{n}\right)\right\| \\
& \geqslant \mid\left\|x_{n}-x^{*}\right\|-\left\|\beta_{n}\left(x_{n}-S x_{n}\right)-c_{n}\left(u_{n}-S x_{n}\right)\right\| \|
\end{aligned}
$$

so that

$$
\begin{array}{r}
\left\|y_{n}-x^{*}\right\|^{2} \geqslant\left\|x_{n}-x^{*}\right\|^{2}-2\left\|x_{n}-x^{*}\right\|\left\|\beta_{n}\left(x_{n}-S x_{n}\right)-c_{n}\left(u_{n}-S x_{n}\right)\right\| \\
+\left\|\beta_{n}\left(x_{n}-S x_{n}\right)-c_{n}\left(u_{n}-S x_{n}\right)\right\|^{2} .
\end{array}
$$

Using (2.10) in (2.9) yields

$$
\begin{aligned}
& \left\langle y_{n}-S y_{n}, j\left(x_{n}-x^{*}\right)\right\rangle \\
& \geqslant r\left(y_{n}, x^{*}\right)\left\|x_{n}-x^{*}\right\|^{2}-2 r\left(y_{n}, x^{*}\right)\left\|x_{n}-x^{*}\right\|\left\|\beta_{n}\left(x_{n}-S x_{n}\right)-c_{n}\left(u_{n}-S x_{n}\right)\right\| \\
& \quad+r\left(y_{n}, x^{*}\right)\left\|\beta_{n}\left(x_{n}-S x_{n}\right)-c_{n}\left(u_{n}-S x_{n}\right)\right\|^{2} \\
& \quad+\left\langle y_{n}-S y_{n}, j\left(x_{n}-x^{*}\right)-j\left(y_{n}-x^{*}\right),\right.
\end{aligned}
$$


and using this in (2.8) we obtain

$$
\begin{aligned}
& \left\|x_{n+1}-x^{*}\right\|^{2} \\
& \leqslant\left[1-2 \alpha_{n} r\left(y_{n}, x^{*}\right)\right]\left\|x_{n}-x^{*}\right\|^{2}+4 \alpha_{n}\left\|x_{n}-x^{*}\right\|\left\|\beta_{n}\left(x_{n}-S x_{n}\right)-c_{n}\left(u_{n}-S x_{n}\right)\right\| \\
& \quad+2 c_{n} \alpha_{n}\left\langle u_{n}-S x_{n}, j\left(x_{n}-x^{*}\right)\right\rangle+\frac{2 \alpha_{n} d^{*} r}{(\mu+n+1)}+M \alpha_{n} \frac{\rho_{E}\left(d^{*} \alpha_{n}\right)}{\alpha_{n}} \\
& \quad-2 \alpha_{n}\left\langle y_{n}-S y_{n}, j\left(x_{n}-x^{*}\right)-j\left(y_{n}-x^{*}\right)\right\rangle \\
& \leqslant\left[1-2 \alpha_{n} r\left(y_{n}, x^{*}\right)\right]\left\|x_{n}-x^{*}\right\|^{2}+4 \alpha_{n} \beta_{n} d^{*} r+\frac{2 d^{*} r \alpha_{n}}{(\mu+n+1)}+\frac{2 d^{*} r \alpha_{n}}{(\mu+n+1)} \\
& \quad+M \alpha_{n} \frac{\rho_{E}\left(d^{*} \alpha_{n}\right)}{\alpha_{n}}+2 d \alpha_{n}\left\|j\left(x_{n}-x^{*}\right)-j\left(y_{n}-x^{*}\right)\right\| \\
& \leqslant\left[1-2 \alpha_{n} r\left(y_{n}, x^{*}\right)\right]\left\|x_{n}-x^{*}\right\|^{2}+\frac{8 \alpha_{n} d^{*} r}{(\mu+n)} \\
& \quad+M \alpha_{n} \frac{\rho_{E}\left(d^{*} \alpha_{n}\right)}{\alpha_{n}}+2 d \alpha_{n}\left\|j\left(x_{n}-x^{*}\right)-j\left(y_{n}-x^{*}\right)\right\| .
\end{aligned}
$$

Hence

$$
\begin{aligned}
r^{2}<\left\|x_{n+1}-x^{*}\right\|^{2} & \leqslant\left[1-\alpha_{n} r\left(y_{n}, x^{*}\right)\right] r^{2}+\frac{8 d^{*} r \alpha_{n}}{\mu}+M \alpha_{n} \frac{\rho_{E}\left(d^{*} \alpha_{n}\right)}{\alpha_{n}} \\
& +2 d \alpha_{n}\left\|j\left(x_{n}-x^{*}\right)-j\left(y_{n}-x^{*}\right)\right\| \\
& \leqslant r^{2}-\alpha_{n}\left[\frac{\phi\left(\frac{1}{2} r\right) r^{2}}{(1+\phi(r)+r)}-\frac{8 d^{*} r}{\mu}-M \frac{\rho_{E}\left(d^{*} \alpha_{n}\right)}{\alpha_{n}}\right. \\
& \left.-2 d\left\|j\left(x_{n}-x^{*}\right)-j\left(y_{n}-x^{*}\right)\right\|\right] \leqslant r^{2},
\end{aligned}
$$

a contradiction, so that $x_{n+1} \in B$. Hence $x_{n} \in B, \forall n \geqslant 0$.

We now prove that $\lim _{n \rightarrow \infty} x_{n}=x^{*}$. From (2.12) we obtain

$$
\begin{aligned}
\left\|x_{n+1}-x^{*}\right\|^{2} & \leqslant\left[1-2 \alpha_{n} r\left(y_{n}, x^{*}\right)\right]\left\|x_{n}-x^{*}\right\|^{2}+\frac{8 d^{*} r \alpha_{n}}{(\mu+n)} \\
& +2 \alpha_{n} d\left\|j\left(x_{n}-x^{*}\right)-j\left(y_{n}-x^{*}\right)\right\|+M \frac{\alpha_{n} \rho_{E}\left(d^{*} \alpha_{n}\right)}{\alpha_{n}} \\
& =\left[1-2 \alpha_{n} r\left(y_{n}, x^{*}\right)\right]\left\|x_{n}-x^{*}\right\|^{2}+\alpha_{n} \lambda_{n}
\end{aligned}
$$

where

$$
\lambda_{n}=\frac{8 d^{*} r}{(\mu+n)}+2 d\left\|j\left(x_{n}-x^{*}\right)-j\left(y_{n}-x^{*}\right)\right\|+M \frac{\rho_{E}\left(d^{*} \alpha_{n}\right)}{\alpha_{n}} .
$$

Observe that $\lim \inf \left\|y_{n}-x^{*}\right\|=\liminf \left\|x_{n}-x^{*}\right\|$. Let $\liminf \left\|y_{n}-x^{*}\right\|=\delta \geqslant 0$. We prove that $\delta=0$. Assume for contraction that $\delta>0$. Then there exists a positive integer $N_{0}$ such that $\left\|y_{n}-x^{*}\right\| \geqslant \frac{1}{2} \delta$ and $\left\|x_{n}-x^{*}\right\| \geqslant \frac{1}{2} \delta, \forall n \geqslant N_{0}$. Since $\lim \lambda_{n}=0$, we can choose a positive integer $N_{1}$ such that

$$
\lambda_{n} \leqslant \frac{\delta^{2} \phi\left(\frac{1}{2} \delta\right)}{4(1+\phi(r)+r)}, \quad \forall n \geqslant N_{1} .
$$


Hence, for all $n \geqslant N=\max \left\{N_{0}, N_{1}\right\}$ we have

$$
\left\|x_{n+1}-x^{*}\right\|^{2} \leqslant\left\|x_{n}-x^{*}\right\|^{2}-\frac{\alpha_{n} \delta^{2} \phi\left(\frac{1}{2} \delta\right)}{4(1+\phi(r)+r)}-\alpha_{n}\left[\frac{\delta^{2} \phi\left(\frac{1}{2} \delta\right)}{4(1+\phi(r)+r)}-\lambda_{n}\right],
$$

so that

$$
\frac{\delta^{2} \phi\left(\frac{1}{2} \delta\right)}{4(1+\phi(r)+r)} \alpha_{n} \leqslant\left\|x_{n}-x^{*}\right\|^{2}-\left\|x_{n+1}-x^{*}\right\|^{2}, \quad \forall n \geqslant N
$$

Thus

$$
\frac{\delta^{2} \phi\left(\frac{1}{2} \delta\right)}{4(1+\phi(r)+r)} \sum_{j=N}^{n} \alpha_{j} \leqslant\left\|x_{N}-x^{*}\right\|,
$$

so that $\sum_{n=0}^{\infty} \alpha_{n}<\infty$ contradicting $\sum_{n=0}^{\infty} \alpha_{n}=\infty$.

Hence $\liminf \left\|y_{n}-x^{*}\right\|=\liminf \left\|x_{n}-x^{*}\right\|=0$, so that there exists a subsequence $\left\{\left\|x_{n_{j}}-x^{*}\right\|\right\}_{n=0}^{\infty}$ of the sequence $\left\{\left\|x_{n}-x^{*}\right\|\right\}$ such that $\lim _{j \rightarrow \infty}\left\|x_{n_{j}}-x^{*}\right\|=0$. It follows that given any $\epsilon>0$ there exists a positive integer $j_{0}$ such that

$$
\left\|x_{n_{j}}-x^{*}\right\|<\epsilon, \quad \forall j \geqslant j_{0} \quad\left(\forall n_{j} \geqslant n_{j_{0}}\right) .
$$

Since $\lim \lambda_{n}=\lim \alpha_{n}=\lim \beta_{n}=0$, there exists a positive integer $N_{2}$ such that

$$
\lambda_{n} \leqslant \frac{2 \epsilon^{2} \phi\left(\frac{1}{2} \epsilon\right)}{(1+\phi(r)+r)}, \quad \alpha_{n} \leqslant \frac{\epsilon}{8 d^{*}}, \quad \beta_{n} \leqslant \frac{\epsilon}{8 d^{*}} \quad \forall n \geqslant N_{2} .
$$

Since $\lim _{j \rightarrow \infty} n_{j}=\infty$, we can choose $j_{*}$ such that $n_{j_{*}} \geqslant \max \left\{n_{j_{0}}, N_{2}\right\}$ so that $\| x_{n_{j_{*}}}-$ $x^{*} \|<\epsilon$, and

$$
\lambda_{n} \leqslant \frac{2 \epsilon^{2} \phi\left(\frac{1}{2} \epsilon\right)}{(1+\phi(r)+r)}, \quad \alpha_{n} \leqslant \frac{\epsilon}{8 d^{*}}, \quad \beta_{n} \leqslant \frac{\epsilon}{8 d^{*}} \quad \forall n \geqslant n_{j_{*}} .
$$

We prove that

$$
\left\|x_{n_{j_{*}}+p}-x^{*}\right\|<\epsilon, \text { for all integers } p \geqslant 1 .
$$

The proof is by induction. For $p=1$, we prove that $\left\|x_{n_{j_{*}}+1}-x^{*}\right\|<\epsilon$. Suppose for contradiction that $\left\|x_{n_{j_{*}}+1}-x^{*}\right\| \geqslant \epsilon$. Then

$\left\|x_{n_{j_{*}}}-x^{*}\right\| \geqslant\left\|x_{n_{j_{*}}+1}-x^{*}\right\|-\alpha_{n_{j_{*}}}\left\|x_{n_{j_{*}}}-S y_{n_{j_{*}}}\right\|-c_{n_{j_{*}}}^{\prime}\left\|v_{n_{j_{*}}}-S y_{n_{j_{*}}}\right\| \geqslant \epsilon-2 \alpha_{n_{j_{*}}} d^{*} \geqslant \frac{3}{4} \epsilon$,

and

$\left\|y_{n_{j_{*}}}-x^{*}\right\| \geqslant\left\|x_{n_{j_{*}}}-x^{*}\right\|-\beta_{n_{j_{*}}}\left\|x_{n_{j_{*}}}-S x_{n_{j_{*}}}\right\|-c_{n_{j_{*}}}\left\|u_{n_{j_{*}}}-S x_{n_{j_{*}}}\right\| \geqslant \frac{3}{4} \epsilon-2 d^{*} \beta_{n_{j_{*}}} \geqslant \frac{1}{2} \epsilon$.

Hence

$$
r\left(y_{n_{j_{*}}}, x^{*}\right) \geqslant \frac{\phi\left(\frac{1}{2} \epsilon\right)}{(1+\phi(r)+r)},
$$

so that it follows from (2.13) that

$$
\epsilon^{2} \leqslant\left\|x_{n_{j_{*}}+1}-x^{*}\right\|^{2}<\epsilon^{2}-2 \alpha_{n_{j_{*}}} r\left(y_{n_{j_{*}}}, x^{*}\right) \epsilon^{2}+\alpha_{n_{j_{*}}} \lambda_{n_{j_{*}}} \leqslant \epsilon^{2},
$$


a contradiction, so that $\left\|x_{n_{j_{*}+1}}-x^{*}\right\|<\epsilon$. Assume now that $\left\|x_{n_{j_{*}}+p_{0}}-x^{*}\right\|<\epsilon$ for some $p_{0}>1$. We prove that $\left\|x_{n_{j_{*}}}+\left(p_{0}+1\right)-x^{*}\right\|<\epsilon$. Assume for contradiction that $\left\|x_{n_{j_{*}}+\left(p_{0}+1\right)}-x^{*}\right\| \geqslant \epsilon$. Then

$$
\begin{aligned}
\left\|x_{n_{j_{*}}+p_{0}}-x^{*}\right\| \geqslant\left\|x_{n_{j_{*}}+\left(p_{0}+1\right)}-x^{*}\right\|-\alpha_{n_{j_{*}}+p_{0}}\left\|x_{n_{j_{*}}+p_{0}}-S y_{n_{j_{*}}+p_{0}}\right\| & \\
& -c_{n_{j_{*}}+p_{0}}^{\prime}\left\|v_{n_{j_{*}}+p_{0}}-S y_{n_{j_{*}}+p_{0}}\right\| \geqslant \frac{3}{4} \epsilon,
\end{aligned}
$$

and

$$
\begin{gathered}
\left\|y_{n_{j_{*}}+p_{0}}-x^{*}\right\| \geqslant\left\|x_{n_{j_{*}}+p_{0}}-x^{*}\right\|-\beta_{n_{j_{*}}+p_{0}}\left\|x_{n_{j_{*}}+p_{0}}-S x_{n_{j_{*}}+p_{0}}\right\| \\
\quad-c_{n_{j_{*}}+p_{0}}\left\|u_{n_{j_{*}}+p_{0}}-S x_{n_{j_{*}}+p_{0}}\right\| \\
\geqslant \frac{3}{4} \epsilon-2 \beta_{n_{j_{*}}+p_{0}} d^{*} \geqslant \frac{1}{2} \epsilon .
\end{gathered}
$$

From (2.13) we obtain

$$
\epsilon^{2} \leqslant\left\|x_{n_{j_{*}}+\left(p_{0}+1\right)}-x^{*}\right\|^{2}<\epsilon^{2}-2 \epsilon^{2} \alpha_{n_{j_{*}}+p_{0}} r\left(y_{n_{j_{*}}+p_{0}}, x^{*}\right)+\alpha_{n_{j_{*}}+p_{0}} \lambda_{n_{j_{*}}+p_{0}} \leqslant \epsilon^{2},
$$

a contraction, so that $\left\|x_{n_{j_{*}}+\left(p_{0}+1\right)}-x^{*}\right\|<\epsilon$. Hence $\left\|x_{n_{j_{*}}+p}-x^{*}\right\|<\epsilon$, for all integers $p \geqslant 1$, and this implies $\lim \left\|x_{n}-x^{*}\right\|=0$, completing the proof of Theorem 2.1.

\subsection{Convergence theorems for $m$-accretive operator equations}

Corollary 2.2. Let $E$ be a real uniformly smooth Banach space and let $T: D(T) \subseteq$ $E \rightarrow E$ be an $m$-accretive operator with open domain $D(T)$. Let $x^{*}$ denote the solution of the equation $x+T x=f, f \in E$. Define $S: D(T) \rightarrow E$ by $S x=f-T x$. Let $\left\{a_{n}\right\}$, $\left\{b_{n}\right\},\left\{c_{n}\right\},\left\{a_{n}^{\prime}\right\},\left\{b_{n}^{\prime}\right\},\left\{c_{n}^{\prime}\right\},\left\{u_{n}\right\}$ and $\left\{v_{n}\right\}$ be as in Theorem 2.1. Then there exist a real number $\mu \geqslant 1$ and a neighbourhood $B \subseteq D(T)$ of $x^{*}$ such that starting with arbitrary $x_{0}, u_{0}, v_{0} \in B$, the sequence $\left\{x_{n}\right\}_{n=0}^{\infty}$ defined iteratively by

$$
\begin{aligned}
y_{n} & =a_{n} x_{n}+b_{n} S x_{n}+c_{n} u_{n}, \quad n \geqslant 0, \\
x_{n+1} & =a_{n}^{\prime} x_{n}+b_{n}^{\prime} S y_{n}+c_{n}^{\prime} v_{n}, \quad n \geqslant 0,
\end{aligned}
$$

remains in $B$ and converges strongly to $x^{*}$.

Proof. As in the proof of Theorem 2.1, set $\beta_{n}=b_{n}+c_{n}$, and $\alpha_{n}=b_{n}^{\prime}+c_{n}^{\prime}$, then

$$
\begin{aligned}
y_{n}=\left(1-\beta_{n}\right) x_{n}+\beta_{n} S x_{n}+c_{n}\left(u_{n}-S x_{n}\right), & n \geqslant 0, \\
x_{n+1} & =\left(1-\alpha_{n}\right) x_{n}+\alpha_{n} S y_{n}+c_{n}^{\prime}\left(v_{n}-S y_{n}\right), \quad n \geqslant 0 .
\end{aligned}
$$

Observe that $x^{*}$ is a fixed point of $S$, and, since $T$ is accretive, $(I-S)$ is also accretive. The corollary follows from Theorem 2.1.

\subsection{Convergence theorems for $\Phi$-strong pseudocontractions}

Corollary 2.3. Let $E$ be a real uniformly smooth Banach space and let $T: D(T) \subseteq$ $E \rightarrow E$ be a $\phi$-strong pseudocontraction with open domain $D(T) \subseteq E$. Suppose $T$ has 
a fixed point $x^{*} \in D(T)$. Let $\left\{a_{n}\right\},\left\{b_{n}\right\},\left\{c_{n}\right\},\left\{a_{n}^{\prime}\right\},\left\{b_{n}^{\prime}\right\},\left\{c_{n}^{\prime}\right\},\left\{u_{n}\right\}$ and $\left\{v_{n}\right\}$ be as in Theorem 2.1. Then there exist a real number $\mu \geqslant 1$ and a neighbourhood $B \subseteq D(T)$ of $x^{*}$ such that starting with arbitrary $x_{0}, u_{0}, v_{0} \in B$, the sequence $\left\{x_{n}\right\}_{n=0}^{\infty}$, defined iteratively by

$$
\begin{aligned}
y_{n}=a_{n} x_{n}+b_{n} T x_{n}+c_{n} u_{n}, \quad n \geqslant 0, \\
x_{n+1}=a_{n}^{\prime} x_{n}+b_{n}^{\prime} T y_{n}+c_{n}^{\prime} v_{n}, \quad n \geqslant 0,
\end{aligned}
$$

remains in $B$ and converges strongly to $x^{*}$.

Proof. As in the proof of Theorem 2.1, set $\beta_{n}=b_{n}+c_{n}$, and $\alpha_{n}=b_{n}^{\prime}+c_{n}^{\prime}$, then

$$
\begin{aligned}
y_{n}=\left(1-\beta_{n}\right) x_{n}+\beta_{n} T x_{n}+c_{n}\left(u_{n}-T x_{n}\right), & n \geqslant 0, \\
x_{n+1} & =\left(1-\alpha_{n}\right) x_{n}+\alpha_{n} T y_{n}+c_{n}^{\prime}\left(v_{n}-T y_{n}\right), \quad n \geqslant 0 .
\end{aligned}
$$

Since $T$ is $\phi$-strongly pseudocontractive, $(I-T)$ is accretive. The corollary follows from Theorem 2.1.

\subsection{Convergence theorems for quasi-accretive operators}

An operator $T: D(T) \subseteq E$ is called quasi-accretive if $N(T):=\{x \in D(T): T x=0\} \neq$ $\emptyset$ and for all $x \in D(T)$ and $x^{*} \in N(T)$, there exists $j\left(x-x^{*}\right) \in J\left(x-x^{*}\right)$ such that

$$
\left\langle T x-T x^{*}, j\left(x-x^{*}\right)\right\rangle \geqslant 0,
$$

and is called strongly quasi-accretive if there exists $k>0$ such that

$$
\left\langle T x-T x^{*}, j\left(x-x^{*}\right)\right\rangle \geqslant k\left\|x-x^{*}\right\|^{2} .
$$

$T$ is called $\phi$-strongly quasi-accretive if $N(T) \neq \emptyset$ and for all $x \in D(T)$ and $x^{*} \in N(T)$ there exist $j\left(x-x^{*}\right) \in J\left(x-x^{*}\right)$ and a strictly increasing function $\phi:[0, \infty) \rightarrow[0, \infty)$ with $\phi(0)=0$ such that

$$
\left\langle T x-T x^{*}, j\left(x-x^{*}\right)\right\rangle \geqslant \phi\left(\left\|x-x^{*}\right\|\right)\left\|x-x^{*}\right\| .
$$

It is easy to see that our proof of Theorem 2.1 carries over to the class of $\phi$-strongly quasi-accretive operators, while the proof of Corollary 2.2 carries over to quasi-accretive operators, if the operators are locally bounded at each interior point of their effective domains.

\subsection{Convergence theorems for $\Phi$-hemicontractions}

An operator $T: D(T) \subseteq E \rightarrow E$ is called a $\phi$-hemicontraction (see, for example, [29]) if $F(T):=\{x \in D(T): x \in T x\} \neq \emptyset$ and for all $x \in D(T), x^{*} \in F(T)$, there exist $j\left(x-x^{*}\right) \in J\left(x-x^{*}\right)$ and a strictly increasing function $\phi:[0, \infty) \rightarrow[0, \infty)$ with $\phi(0)=0$ such that

$$
\left\langle T x-x^{*}, j\left(x-x^{*}\right)\right\rangle \leqslant\left\|x-x^{*}\right\|^{2}-\phi\left(\left\|x-x^{*}\right\|\right)\left\|x-x^{*}\right\| .
$$

An example in [14] shows that the class of $\phi$-strong pseudocontractions with non-empty fixed-point sets is a proper subclass of the class of $\phi$-hemicontractions.

It is easy to see that Corollary 2.3 carries over to $\phi$-hemicontractions $T$ for which $(I-T)$ is locally bounded at each interior point of its effective domain. 
Acknowledgements. This work was done while M.O.O. was visiting the International Centre for Theoretical Physics, Trieste, Italy as an Associate. He is grateful to the Swedish Agency for Research Cooperation with Developing Countries (SAREC) for generous contribution towards the visit. His research was supported by a grant from TWAS (94-224 RG/MATHS/AF/AC).

\section{References}

1. F. E. BRowder, Nonlinear mappings of nonexpansive and accretive type in Banach spaces, Bull. Am. Math. Soc. 73 (1967), 875-882.

2. R. E. BRUCK JR, The iterative solution of the equation $y \in x+T x$ for a monotone operator T in Hilbert space, Bull. Am. Math. Soc. 79 (1973), 1258-1261.

3. J. CARISTI, The fixed point theory for mappings satisfying inwardness conditions, $\mathrm{PhD}$ thesis (The University of Iowa, Iowa City, 1975).

4. S.-S. CHANG, On Chidume's open questions and approximate solutions of multivalued strongly accretive mapping equations in Banach spaces, J. Math. Analysis Appl. 216 (1997), 94-111.

5. C. E. Chidume, Iterative approximation of fixed points of Lipschitz strictly pseudocontractive mappings, Proc. Am. Math. Soc. 99 (1987), 283-288.

6. C. E. Chidume, An iterative process for nonlinear Lipschitzian strongly accretive mappings in $L_{p}$ spaces, J. Math. Analysis Appl. 151 (1990), 453-461.

7. C. E. Chidume, Approximation of fixed points of strongly pseudo-contractive mappings, Proc. Am. Math. Soc. 120 (1994), 545-551.

8. C. E. Chidume, Iterative solution of nonlinear equations with strongly accretive operators, J. Math. Analysis Appl. 192 (1995), 502-518.

9. C. E. Chidume, Iterative solution of nonlinear equations in smooth Banach spaces, Nonlinear Analysis TMA 26 (1996), 1823-1834.

10. C. E. Chidume, Steepest descent method for locally accretive mappings, J. Korean Math. Soc. 33 (1996), 1-14.

11. C. E. Chidume, Iterative solutions of nonlinear equations of the strongly accretive type, Math. Nachr. 189 (1998), 46-60.

12. C. E. Chidume, Global iteration schemes for strongly pseudocontractive maps, Proc. Am. Math. Soc. 126 (1998), 2641-2649.

13. C. E. Chidume and C. Moore, The solution by iteration of nonlinear equations in uniformly smooth Banach spaces, J. Math. Analysis Appl. 215 (1997), 132-146.

14. C. E. Chidume And M. O. Osilike, Fixed point iterations for strictly hemi-contractive maps in uniformly smooth Banach spaces, Numer. Funct. Analysis Optim. 15 (1994), 779-790.

15. C. E. Chidume And M. O. Osilike, Approximation methods for nonlinear operator equations of the $m$-accretive type, J. Math. Analysis Appl. 189 (1995), 225-239.

16. C. E. Chidume And M. O. Osilike, Ishikawa iteration process for nonlinear Lipschitz strongly accretive mappings, J. Math. Analysis Appl. 192 (1995), 727-741.

17. C. E. Chidume and M. O. Osilike, Nonlinear accretive and pseudocontractive operator equations in Banach spaces, Nonlinear Analysis 31 (1998), 779-789.

18. L. Deng, On Chidume's open questions, J. Math. Analysis Appl. 174 (1993), 441-449.

19. L. DENG, Iteration processes for nonlinear Lipschitzian strongly accretive mappings in $L_{p}$ spaces, J. Math. Analysis Appl. 188 (1994), 128-140.

20. L. DENG AND X. P Ding, Iterative approximation of Lipschitz strictly pseudo-contractive mappings in uniformly smooth Banach spaces, Nonlinear Analysis TMA 24 (1995), 981987. 
21. Z. HAIYUN, Iterative solution of nonlinear equations involving strongly accretive operators without the Lipschitz assumption, J. Math. Analysis Appl. 213 (1997), 296-307.

22. Z. Haiyun And J. Yuting, Approximation of fixed points of strongly pseudocontractive maps without Lipschitz assumption, Proc. Am. Math. Soc. 125 (1997), 1705-1709.

23. S. IshikawA, Fixed point by a new iteration method, Proc. Am. Math. Soc. 44 (1974), 147-150.

24. T. KAто, Nonlinear semigroups and evolution equations, J. Math. Soc. Japan 19 (1964), $508-520$.

25. L-S. LIU, Ishikawa and Mann iteration process with errors for nonlinear strongly accretive mappings in Banach spaces, J. Math. Analysis Appl. 194 (1995), 114-125.

26. L.-S. LiU, Approximation of fixed points of a strictly pseudocontractive mapping, Proc. Am. Math. Soc. 125 (1997), 1363-1366.

27. W. R. Mann, Mean value methods in iteration, Proc. Am. Math. Soc. 4 (1953), 506-510.

28. R. H. Martin JR, A global existence theorem for autonomous differential equations in Banach spaces, Proc. Am. Math. Soc. 26 (1970), 307-314.

29. M. O. OsILIKE, Iterative solution of nonlinear equations of the $\phi$-strongly accretive type, J. Math. Analysis Appl. 200 (1996), 259-271.

30. M. O. Osilike, Ishikawa and Mann iteration methods with errors for nonlinear equations of the accretive type, J. Math. Analysis Appl. 213 (1997), 91-105.

31. J. SCHU, Iterative construction of fixed points of strictly pseudocontractive mappings, Applicable Analysis 40 (1991), 67-72.

32. J. Schu, On a theorem of C. E. Chidume concerning the iterative approximation of fixed points, Math. Nachr. 153 (1991), 313-319.

33. K.-K. TAN AND H.-K. XU, Iterative solutions to nonlinear equations of strongly accretive operators in Banach spaces, J. Math. Analysis Appl. 178 (1993), 9-21.

34. M. M. VAinberG, On the convergence of the method of steepest descent for nonlinear equations, Sibirsk Mat. Z. 2 (1961), 201-220.

35. X. L. Weng, Fixed point iteration for local strictly pseudocontractive mappings, Proc. Am. Math. Soc. 113 (1991), 727-731.

36. H. K. XU, Inequalities in Banach spaces with applications, Nonlinear Analysis TMA 16 (1991), 1127-1138.

37. Y. XU, Ishikawa and Mann iteration processes with errors for nonlinear strongly accretive operator equations, J. Math. Analysis Appl. 224 (1998), 91-101.

38. Z. B. XU AND G. F. RoACH, Characteristic inequalities of uniformly convex and uniformly smooth spaces, J. Math. Analysis Appl. 157 (1991), 189-210.

39. Z. B. XU AND G. F. ROACH, A necessary and sufficient condition for convergence of steepest descent approximation to accretive operator equations, J. Math. Analysis Appl. 167 (1992), 340-354.

40. Z. B. XU, B. Zhang AND G. F. RoACH, On the steepest descent approximation to solutions of nonlinear strongly accretive operator equations, J. Comput. Math. Supp. (1992), 173-182.

41. L. ZHU, Iterative solution of nonlinear equations involving $m$-accretive operators in Banach spaces, J. Math. Analysis Appl. 188 (1994), 410-415. 